\title{
A Green's Function Approach for Dynamic Stress Analysis of Spherical Shell under the Isotropic Impact Load
}

\author{
Jincheng Lv, Shike Zhang, and Xinsheng Yuan \\ School of Civil Engineering and Architecture, Anyang Normal University, Anyang, Henan 455000, China \\ Correspondence should be addressed to Shike Zhang; shikezhang1021@163.com
}

Received 23 June 2015; Revised 5 November 2015; Accepted 11 November 2015

Academic Editor: Giovanni Falsone

Copyright (C) 2015 Jincheng Lv et al. This is an open access article distributed under the Creative Commons Attribution License, which permits unrestricted use, distribution, and reproduction in any medium, provided the original work is properly cited.

\begin{abstract}
A Green's function approach is developed for the analytic solution of thick-walled spherical shell under an isotropic impact load, which involves building Green's function of this problem by using the appropriate boundary conditions of thick-walled spherical shell. This method can be used to analyze displacement distribution and dynamic stress distribution of the thick-walled spherical shell. The advantages of this method are able (1) to avoid the superposition process of quasi-static solution and free vibration solution during decomposition of dynamic general solution of dynamics, (2) to well adapt for various initial conditions, and (3) to conveniently analyze the dynamic stress distribution using numerical calculation. Finally, a special case is performed to verify that the proposed Green's function method is able to accurately analyze the dynamic stress distribution of thick-walled spherical shell under an isotropic impact load.
\end{abstract}

\section{Introduction}

In practical engineering, isotropic spherical surfaces or shells have a wide range of uses in thermodynamics analysis, electromagnetic analysis, tectonic dynamics analysis of the earth, and so forth. Since Hu had the pioneering research for the general theory of elasticity of isotropic spherical medium [1], more and more researchers attached importance to the study of isotropic spherical surface problems, and they have made great developments. Isotropic spherical surface or shell problems are investigated mainly through the analytic solution method, test method, and numerical computation method in [2-6].

From mathematics and computational perspective, displacement and stress distribution of isotropic spherical surfaces or shells under the isotropic impact load can be obtained by the method of characteristics, integral transformation method, generalized ray method, approximation method, and domain decomposition method in [7-11]. In the 1990s, tectonic dynamics of the earth was considered as a new subject, and researchers have studied deformation problems of isotropic spherical surfaces and shells with multilayer hybrid model. Here Ding et al. in [12] introduced three displacement functions to motion equations of elasticity problems in spherically isotropic media; this effectively simplifies motion equations and improves the solution method of ordinary differential equations for dynamics problems. An analytic solution concerning elastic spherical media under the impact load was given by using characteristic function expansion method to decompose dynamics into quasi-static elastic solution and free vibration solution at inhomogeneous boundary condition in $[13,14]$. These methods, however, are generally suitable for analytical solutions in simple spherical media, which could lead to difficulties in analyzing displacement and dynamic stress distribution of thick-walled spherical shell under the isotropic impact load, especially when using numerical computation to analyze these problems.

To overcome difficulties mentioned above, Green's function of elastic solid sphere was established to solve spherical surface and shell problems under an isotropic impact load in [15-17]. However, the established analytic solutions and mathematical expression of dynamic stress distribution are not well suitable for the thick-walled spherical shells. 
The objective of this paper is to develop a Green function of the thick-walled spherical shells under an isotropic impact load of solid sphere based on the research ideas of the aforementioned paper and build the mathematical expression of displacement and dynamic stress distribution. The solution of the problem is obtained by calculating Green's function that links the stress distribution to the normal displacements at the interface and, meanwhile, is suitable for various boundary conditions. The advantage of methodology does not need to not only decompose the solution of the problem into quasi-static solution and free vibration solution, but also avoid the complicated integral transformation. It is, therefore, relatively convenient to be conducted by using numerical calculation for analysis of dynamic stress distribution. A case study in [18] is applied to verify the availability and accuracy of the proposed new method. Results illustrate that dynamic stress distributions obtained in this case study are in good agreement with actual data.

\section{Formulation of Green's Function}

For a thick-walled spherical shell, there is a distinct difference between internal radius and external radius. Internal radius and external radius are set to $a$ and $b$, respectively, and are under normal impact loads that are $\psi_{1}(t)$ and $\psi_{2}(t)$, respectively. Due to the spherical symmetry of the problem, the motion equation of the problem in spherical coordinates can be expressed only by radial displacement component $u(r, t)$ and is given by

$$
\frac{\partial^{2} u}{\partial t^{2}}-c^{2} \frac{\partial}{\partial r}\left[\frac{1}{r^{2}} \frac{\partial}{\partial r}\left(r^{2} u\right)\right]=\mathbf{0} \quad a \leq r \leq b, t \geq 0
$$

where $u$ is displacement, $E$ is elastic modulus, $\mu$ is Poisson's ratio, $r$ is radius of spherical shell, $t$ is time, and the longitudinal wave velocity is

$$
c=\sqrt{\frac{E(1-\mu)}{\rho(1+\mu)(1-2 \mu)}} .
$$

Let $\psi$ be the normal impact stress; stresses boundary condition of internal radius and external radius at the thickwalled spherical shell are given by

$$
\begin{aligned}
\sigma_{r r}(a, t) & =\frac{E}{(1+\mu)(1-2 \mu)}\left[(1-\mu) \frac{\partial u}{\partial r}+2 \mu \frac{u}{r}\right]_{r=a} \\
& =\psi_{1}(t), \\
\sigma_{r r}(b, t) & =\frac{E}{(1+\mu)(1-2 \mu)}\left[(1-\mu) \frac{\partial u}{\partial r}+2 \mu \frac{u}{r}\right]_{r=b} \\
& =\psi_{2}(t) .
\end{aligned}
$$

Initial condition of the problem is

$$
\begin{gathered}
u(r, 0)=\varphi_{1}(r), \\
\frac{\partial u(r, 0)}{\partial t}=\varphi_{2}(r) .
\end{gathered}
$$

According to Hooke's law, the relationship between dynamic stress and strain based on polar coordinates can be expressed as

$$
\begin{aligned}
& \sigma_{r r}=\frac{E}{(1+\mu)(1-2 \mu)}\left[(1-\mu) \frac{\partial u}{\partial r}+2 \mu \frac{u}{r}\right], \\
& \sigma_{\theta \theta}=\sigma_{\varphi \varphi}=\frac{E}{(1+\mu)(1-2 \mu)}\left[\mu \frac{\partial u}{\partial r}+\frac{u}{r}\right],
\end{aligned}
$$

where $\sigma_{r \theta}=\sigma_{r \varphi}=\sigma_{\varphi \theta}=0$.

According to (1) and (3), Green's function on problem of determining solution can be defined as the following solution of determining solution problem:

$$
\begin{aligned}
& \left\{\frac{\partial^{2}}{\partial t^{2}}-c^{2} \frac{\partial}{\partial r}\left[\frac{1}{r^{2}} \frac{\partial}{\partial r}\left(r^{2}\right)\right]\right\} G(r, t ; \xi, \tau) \\
& =\delta(r-\xi) \delta(t-\tau), \quad a \leq r, \xi \leq b, t \geq 0, \\
& {\left.\left[(\mathbf{1}-\mu) \frac{\partial}{\partial r}+\frac{2 \mu}{r}\right] G(r, t ; \xi, \tau)\right|_{r=a}=\mathbf{0},} \\
& {\left.\left[(\mathbf{1}-\mu) \frac{\partial}{\partial r}+\frac{2 \mu}{r}\right] G(r, t ; \xi, \tau)\right|_{r=b}=\mathbf{0},} \\
& \left.G(r, t ; \xi, \tau)\right|_{t<\tau}=0, \\
& \left.\frac{\partial G(r, t ; \xi, \tau)}{\partial t}\right|_{t<\tau}=0 .
\end{aligned}
$$

In order to solve above-mentioned equations, it is necessary to introduce two lemmas, which are obtained from impulse principle and in [19], as follows.

Lemma 1 (basic integral formula). Green's function, $G(r, t ; \xi$, $\tau)$, is used to express the solution of determining solution problem about (1) and (3) as follows:

$$
\begin{gathered}
u(r, t)=\int_{a}^{b}\left[\left.\varphi_{\mathbf{2}}(r) G(r, t ; \xi, \tau)\right|_{\tau=\mathbf{0}}\right. \\
\left.-\left.\varphi_{\mathbf{1}}(r) \frac{\partial G(r, t ; \xi, \tau)}{\partial \tau}\right|_{\tau=\mathbf{0}}\right] d \xi+\frac{\mathbf{1}}{\rho}
\end{gathered}
$$

$$
\begin{aligned}
& \cdot \int_{0}^{t}\left[\left.\psi_{2}(\tau) G(r, t ; \xi, \tau)\right|_{\xi=b}\right. \\
& \left.-\left.\psi_{1}(\tau) G(r, t ; \xi, \tau)\right|_{\xi=a}\right] d \tau .
\end{aligned}
$$


Lemma 2 (impulse principle). The solution of determining solution problem about (6) is given by

$$
\begin{aligned}
\left\{\frac{\partial^{2}}{\partial t^{2}}-c^{2} \frac{\partial}{\partial r}\left[\frac{1}{r^{2}} \frac{\partial}{\partial r}\left(r^{2}\right)\right]\right\} G(r, t ; \xi, \tau) & =0, \\
a \leq r, \xi \leq b, t \geq 0 & \leq \\
{\left.\left[(\mathbf{1}-\mu) \frac{\partial}{\partial r}+\frac{2 \mu}{r}\right] G(r, t ; \xi, \tau)\right|_{r=a} } & =\mathbf{0}, \\
{\left.\left[(\mathbf{1}-\mu) \frac{\partial}{\partial r}+\frac{2 \mu}{r}\right] G(r, t ; \xi, \tau)\right|_{r=b} } & =\mathbf{0}, \\
\left.G(r, t ; \xi, \tau)\right|_{t=\tau} & =0 \\
\left.\frac{\partial G(r, t ; \xi, \tau)}{\partial t}\right|_{t=\tau} & =\delta(r-\xi) .
\end{aligned}
$$

\section{Solution of Green's Function with a Separation Variable Method}

It can be seen from Lemmas 1 and 2 so long as Green's function $G(r, t ; \xi, \tau)$ is obtained that the solution, $u(r, t)$, about determining solution problem of (1) and (3), can be easily found. In the meantime, the determining solution problem of (8) and boundary conditions are all due to being homogeneous; they are straightforward to be found by the separation variable method.

Let $T=t-\tau$; the determining solution problem of (8) can be rewritten by

$$
\begin{aligned}
& \frac{\partial^{2} G}{\partial T^{2}}-c^{2} \frac{\partial}{\partial r}\left[\frac{1}{r^{2}} \frac{\partial}{\partial r}\left(r^{2} G\right)\right]=0, \\
& {\left.\left[(\mathbf{1}-\mu) \frac{\partial G}{\partial r}+\frac{2 \mu}{r} G\right]\right|_{r=a}=\mathbf{0}, \quad} \\
& {\left.\left[(\mathbf{1}-\mu) \frac{\partial G}{\partial r}+\frac{2 \mu}{r} G\right]\right|_{r=b}=\mathbf{0}, t \geq 0} \\
& \left.G\right|_{T=0}=0, \\
& \left.\frac{\partial G}{\partial t}\right|_{T=0}=\delta(r-\xi) . \\
& \text { Let } G(r, t ; \xi, \tau)=R(r ; \xi, \tau) X(t ; \xi, \tau) ; \text { we get } \\
& \frac{d^{2} X}{d T^{2}}+(\lambda c)^{2} X=0
\end{aligned}
$$

and eigenvalue problem

$$
\begin{aligned}
\frac{d^{2} R}{d r^{2}}+\frac{2}{r} \frac{d R}{d r}+\left(\lambda^{2}-\frac{2}{r^{2}}\right) R & =0 \\
\left(\frac{d R}{d r}+\frac{h}{a} R\right)_{r=a} & =\mathbf{0}, \\
\left(\frac{d R}{d r}+\frac{h}{b} R\right)_{r=b} & =\mathbf{0},
\end{aligned}
$$

where

$$
h=\frac{2 \mu}{(1-\mu)} .
$$

Let

$$
\begin{aligned}
& j_{a}=\lambda j_{1}^{\prime}(\lambda a)+\frac{h}{a} j_{1}(\lambda a), \\
& n_{a}=\lambda n_{1}^{\prime}(\lambda a)+\frac{h}{a} n_{1}(\lambda a), \\
& j_{b}=\lambda j_{1}^{\prime}(\lambda b)+\frac{h}{b} j_{1}(\lambda b), \\
& n_{b}=\lambda n_{1}^{\prime}(\lambda b)+\frac{h}{b} n_{1}(\lambda b) .
\end{aligned}
$$

Characteristic equation and eigenfunction are, respectively,

$$
\begin{aligned}
j_{a} n_{b}-j_{b} n_{a} & =0, \\
R(r ; \xi, \tau) & =n_{a} j_{\mathbf{1}}\left(\lambda_{i} r\right)-j_{a} n_{\mathbf{1}}\left(\lambda_{i} r\right) .
\end{aligned}
$$

Let

$$
\begin{aligned}
& J_{a}=(h+1) J_{1 / 2}(\lambda a)+(h-2) J_{5 / 2}(\lambda a), \\
& Y_{a}=(h+1) Y_{1 / 2}(\lambda a)+(h-2) Y_{5 / 2}(\lambda a), \\
& J_{b}=(h+1) J_{1 / 2}(\lambda b)+(h-2) J_{5 / 2}(\lambda b), \\
& Y_{b}=(h+1) Y_{1 / 2}(\lambda b)+(h-2) Y_{5 / 2}(\lambda b) .
\end{aligned}
$$

We get

$$
\begin{aligned}
& j_{a}=\sqrt{\frac{\pi}{2 \lambda a}} \frac{\lambda}{3} J_{a}, \\
& j_{b}=\sqrt{\frac{\pi}{2 \lambda b}} \frac{\lambda}{3} J_{b}, \\
& n_{a}=\sqrt{\frac{\pi}{2 \lambda a}} \frac{\lambda}{3} Y_{a}, \\
& n_{b}=\sqrt{\frac{\pi}{2 \lambda b}} \frac{\lambda}{3} Y_{b} .
\end{aligned}
$$

Based on the relationship between spherical Bessel function and semiodd order function, the characteristic equation (15) can be rewritten by

$$
J_{a} Y_{b}-J_{b} Y_{a}=\mathbf{0} .
$$

Furthermore, let

$$
M_{r}=\sqrt{\frac{\pi}{2 \lambda r}}\left[Y_{a} J_{3 / 2}(\lambda r)-J_{a} Y_{3 / 2}(\lambda r)\right] .
$$

The eigenfunction (12) is

$$
R=\frac{\lambda}{3} \sqrt{\frac{\pi}{2 \lambda a}} M_{r}
$$


and its 2-norm is

$$
\begin{aligned}
\|R\|^{2} & =\int_{a}^{b} r^{2} R^{2}(r ; \xi, \tau) d r \\
& =\int_{a}^{b} r^{2}\left[n_{a} j_{1}(\lambda r)-j_{a} n_{\mathbf{1}}(\lambda r)\right] d r, \\
\|R\|^{2} & =\frac{N}{\mathbf{8} \lambda^{2} a},
\end{aligned}
$$

where

$$
\begin{aligned}
N= & \frac{(2 h-1)^{2}+4 \lambda^{2} b^{2}-9}{\lambda^{2} b^{2}}\left(\frac{J_{a}}{J_{b}}\right)^{2} \\
& -\frac{(2 h-1)^{2}+4 \lambda^{2} a^{2}-9}{\lambda^{2} a^{2}} .
\end{aligned}
$$

According to principle of linear superposition and orthogonality of Bessel function, we get

$$
G(r, t, \xi, \tau)=\sum_{i=1}^{\infty} C R \sin \lambda_{i} c(t-\tau),
$$

where

$$
C=\frac{\int_{a}^{b} r^{2} R_{i}(r ; \xi, \tau) \delta(r-\xi) d r}{\lambda_{i} c\left\|R_{1, i}\right\|^{2}} .
$$

Let $M_{a}=\left.M_{r}\right|_{r=a}, M_{b}=\left.M_{r}\right|_{r=b}$, and let the thick-walled shell be in a static state when $t=0$; we get

$$
\begin{aligned}
u(r, 0) & =\varphi_{1}(r)=0, \\
\frac{\partial u(r, 0)}{\partial t} & =\varphi_{2}(r)=0 .
\end{aligned}
$$

In the light of Lemma 1, displacement distribution of the thick-walled shell (7) can be expressed by

$$
\begin{aligned}
& u(r, t)=\frac{4 \pi}{9 \rho c} \sum_{i=1}^{\infty} \frac{M_{r}}{N} \\
& \cdot \int_{0}^{t}\left[\lambda_{i}^{2} b^{2} \psi_{2}(t) M_{b}-\lambda_{i}^{2} a^{2} \psi_{1}(t) M_{a}\right] \\
& \cdot \sin \lambda_{i} c(t-\tau) d \tau .
\end{aligned}
$$

\section{Numerical Example}

Case 1 (isotropic impact load on spherical external surface). Let dimensionless variables be $T=(c / a) t, R=(r-a) /(b-a)$, and $\overline{\sigma_{i}}=\sigma_{i} / \sigma_{0}$, assuming $s=b / a$, which is diameter ratio of the thick-walled shell. Function of isotropic impact load with time is

$$
\begin{aligned}
& \psi_{2}(t)=-\sigma_{0} H(t)= \begin{cases}0 & t<0 \\
-\sigma_{0} & t \geq 0^{+}\end{cases} \\
& \psi_{1}(t)=0 .
\end{aligned}
$$

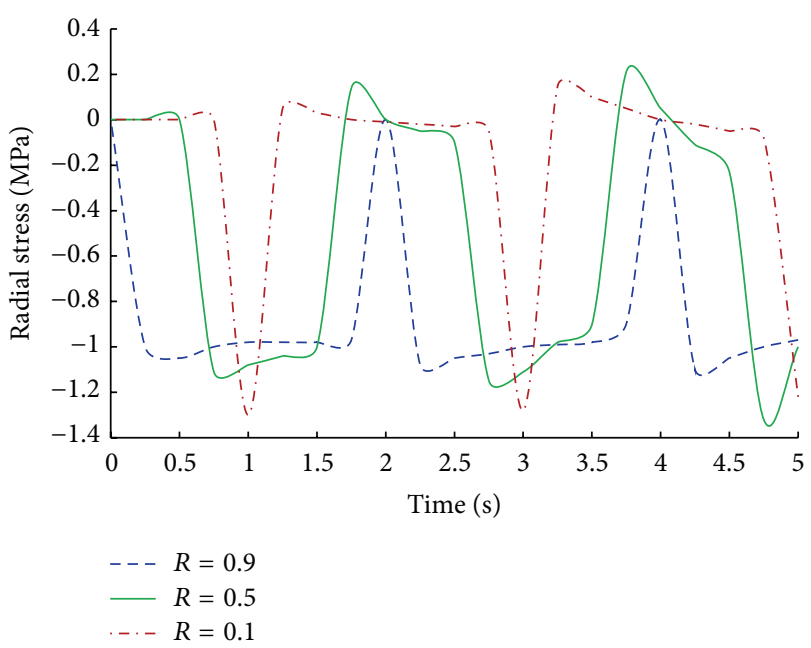

FIGURE 1: Impact radial stress variations of external wall with time $(s=2)$.

Substituting (20) and (21) into (7), the analytic solution of displacement distribution is given by

$$
u(r, t)=\frac{4 \pi \sigma_{0}}{9 \rho c^{2}} \sum_{i=1}^{\infty} \frac{M_{r}}{\lambda_{i} N} \lambda_{i}^{2} b^{2} M_{b}\left(\cos \lambda_{i} c t-1\right) .
$$

According to Bessel function, dynamic stress expression can be changed to

$$
\begin{aligned}
\sigma_{r r} & =\frac{4 \pi \sigma_{0}}{27(1-\mu)} \sum_{i=1}^{\infty} \frac{\lambda_{i}^{2} b^{2}}{N} \sqrt{\frac{\pi}{2 \lambda_{i} r}} M_{b} \\
& \cdot\left\{\begin{array}{c}
(1+\mu)\left[Y_{a} J_{1 / 2}\left(\lambda_{i} r\right)-J_{a} Y_{1 / 2}\left(\lambda_{i} r\right)\right] \\
-(2-4 \mu)\left[Y_{a} J_{5 / 2}\left(\lambda_{i} r\right)-J_{a} Y_{5 / 2}\left(\lambda_{i} r\right)\right]
\end{array}\right\}\left(\cos \lambda_{i} c t\right. \\
& -\mathbf{1}), \\
\sigma_{\theta \theta} & =\frac{4 \pi \sigma_{0}}{27(1-\mu)} \sum_{i=1}^{\infty} \frac{\lambda_{i}^{2} b^{2}}{N} \sqrt{\frac{\pi}{2 \lambda_{i} r}} M_{b} \\
& \cdot\left\{\begin{array}{c}
(1+\mu)\left[Y_{a} J_{1 / 2}\left(\lambda_{i} r\right)-J_{a} Y_{1 / 2}\left(\lambda_{i} r\right)\right] \\
+(1-2 \mu)\left[Y_{a} J_{5 / 2}\left(\lambda_{i} r\right)-J_{a} Y_{5 / 2}\left(\lambda_{i} r\right)\right]
\end{array}\right\}\left(\cos \lambda_{i} c t\right. \\
- & \mathbf{1}) .
\end{aligned}
$$

Assume material properties of isotropic spherical surfaces and shells are obtained: $\mu=0.25, E=200 \mathrm{GPa}$, and $\rho=$ $7.8 \times 10^{3} \mathrm{~kg} / \mathrm{m}^{3}$. Dynamic stress distribution can be obtained by using the proposed Greens function method.

Figure 1 shows radial stress variations with time when the external wall of spherical shell is impacted by instantaneous impact at $s=2$. As shown in Figure 1, there are four various dynamic stress states in three different spread stages of stress wave head. First, the dynamic stress value is zero, when the stress wave head is propagating from one impact 


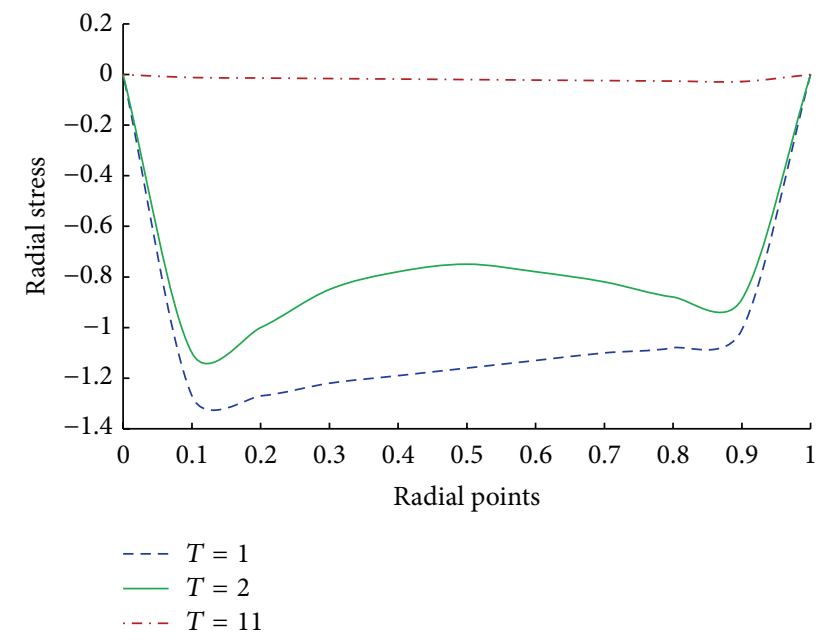

FIGURE 2: Impact radial stress variations of external wall with radius $(s=2)$.

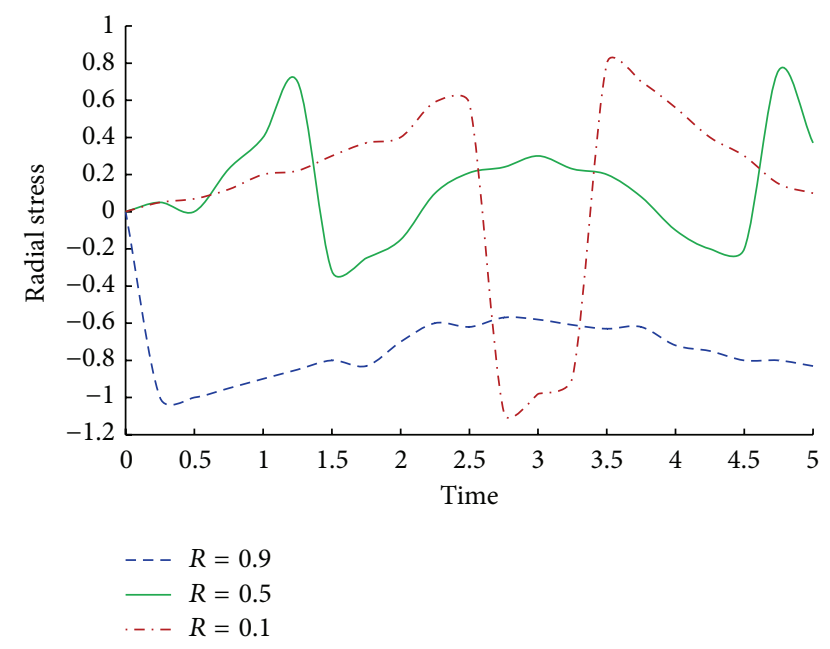

FIGURE 3: Impact radial stress variations of external wall with time $(s=4)$.

wall to the other side, but it did not reach a point of the other side. Second, the dynamic stress value appears as jump discontinuity when the stress wave head has reached points of the other side from one impact wall. Third, the dynamic stress value gently tends to the static stress value under the static load when not only has the stress wave head reached points of the other side from one impact wall, but also it has been far away points of the other side. Last, due to reflection of inner wall, the stress wave presents tensile phenomenon and then rapidly tends to the static stress value under the static load; once the next stress comes to the wall, the dynamic stress value appears as strong jump discontinuity again.

Figure 2 shows the radial stress variations along radial points when the external wall of spherical shell is impacted by instantaneous impact at $s=2$. It can be seen from the

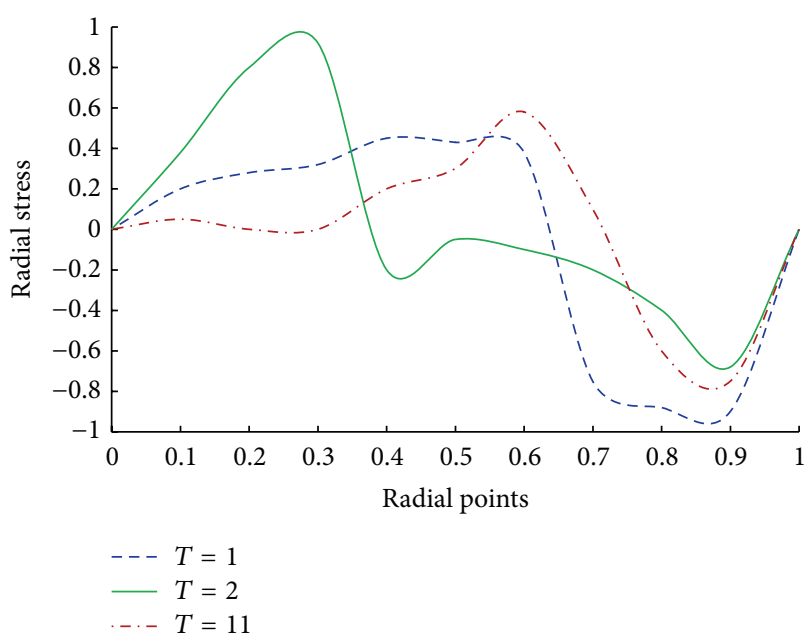

FIGURE 4: Impact radial stress variations of external wall with radius $(s=4)$.

figure that the radial dynamic stress values present significant change in the internal and external wall points, and the maximum dynamic stress near the inner wall.

Figures 3 and 4 show the radial stress variations with time and the radial stress variations along radial points, respectively, when the external wall of spherical shell is impacted by instantaneous impact at $s=4$. As shown in Figures 3 and 4 , with the increase of the cylinder thickness, the radial dynamic stress is first slow tension; second it presents jump with reach of stress wave head; third static stress value will have time extension while the time of reflection wave of the inner wall is lengthened; at last, the maximum compressive stress occurs near the impact wall, while the maximum tensile stress is near the other side.

Results from Figures 1, 2, 3, and 4 verify that the solutions obtained by using the proposed method are accurate in [12, $18,20]$.

Case 2 (isotropic impact load on spherical internal surface). Likewise, function of sudden stress concerning spherical internal surface with time is expressed as follows:

$$
\begin{aligned}
& \psi_{1}(t)=-\sigma_{0} H(t)= \begin{cases}0 & t<0 \\
-\sigma_{0} & t \geq 0^{+}\end{cases} \\
& \psi_{2}(t)=0 .
\end{aligned}
$$

The solution of displacement distribution is given by

$$
u(r, t)=\frac{\mathbf{4} \pi \sigma_{0}}{\mathbf{9} \rho c^{2}} \sum_{i=1}^{\infty} \frac{M_{r}}{\lambda_{i} N} \lambda_{i}^{2} a^{2} M_{a}\left(1-\cos \lambda_{i} c t\right) .
$$




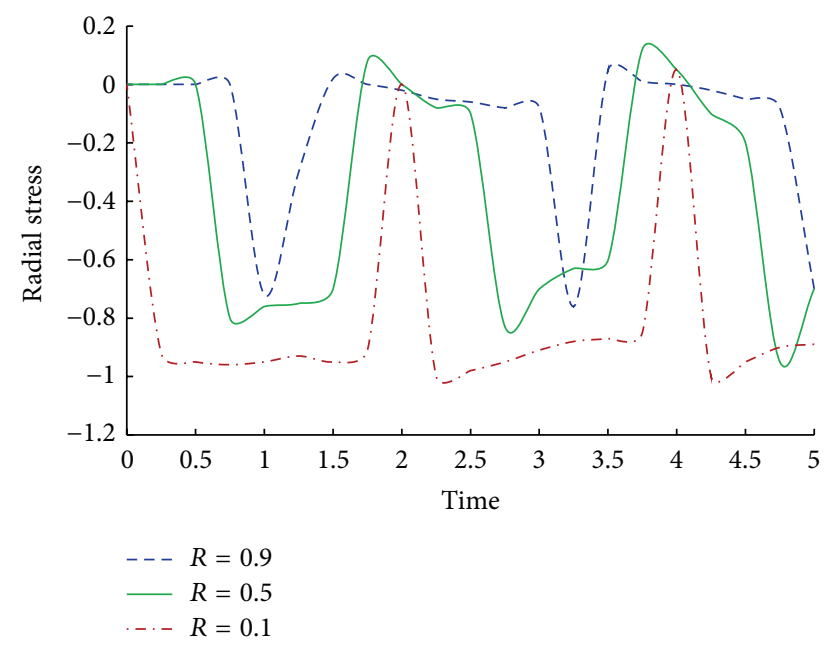

FIGURE 5: Impact radial stress variations of external wall with time $(s=2)$.

Using property of Bessel function, dynamic stress expression can be changed to

$$
\begin{aligned}
\sigma_{r r} & =\frac{4 \pi \sigma_{0}}{27(1-\mu)} \sum_{i=1}^{\infty} \frac{\lambda_{i}^{2} a^{2}}{N} \sqrt{\frac{\pi}{2 \lambda_{i} r}} M_{a} \\
& \cdot\left\{\begin{array}{c}
(1+\mu)\left[Y_{a} J_{1 / 2}\left(\lambda_{i} r\right)-J_{a} Y_{1 / 2}\left(\lambda_{i} r\right)\right] \\
-(2-4 \mu)\left[Y_{a} J_{5 / 2}\left(\lambda_{i} r\right)-J_{a} Y_{5 / 2}\left(\lambda_{i} r\right)\right]
\end{array}\right\}(1 \\
& \left.-\cos \lambda_{i} c t\right), \\
\sigma_{\theta \theta} & =\frac{4 \pi \sigma_{0}}{27(1-\mu)} \sum_{i=1}^{\infty} \frac{\lambda_{i}^{2} a^{2}}{N} \sqrt{\frac{\pi}{2 \lambda_{i} r}} M_{a} \\
& \cdot\left\{\begin{array}{c}
(1+\mu)\left[Y_{a} J_{1 / 2}\left(\lambda_{i} r\right)-J_{a} Y_{1 / 2}\left(\lambda_{i} r\right)\right] \\
+(1-2 \mu)\left[Y_{a} J_{5 / 2}\left(\lambda_{i} r\right)-J_{a} Y_{5 / 2}\left(\lambda_{i} r\right)\right]
\end{array}\right\}(1 \\
& \left.-\cos \lambda_{i} c t\right) .
\end{aligned}
$$

By using material properties of Case 1, dynamic stress distribution can be obtained. For $s=2$, when the internal wall of spherical shell is impacted by instantaneous impact, the radial stress variations with time are shown in Figure 5, and the radial stress variations along radial points are shown in Figure 6. For $s=4$, when the internal wall of spherical shell is impacted by instantaneous impact, the radial stress variations with time are shown in Figure 7, and the radial stress variations along radial points are shown in Figure 8.

As shown in Figures 5, 6, 7, and 8, when the internal wall of spherical shell is impacted by instantaneous impact, results of radial stress distribution with time and radius have similar results that are obtained when the external wall of spherical shell is impacted by instantaneous impact. The detailed analysis can be found in Case 1 . The difference is that when the cylinder is thin, the maximum compression stress is always near the inner wall.

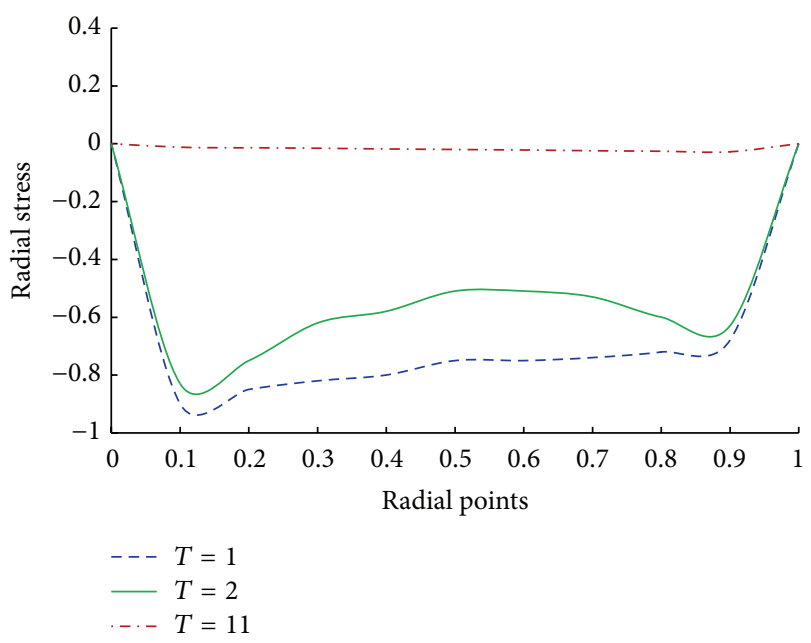

FIGURE 6: Impact radial stress variations of external wall with radius $(s=2)$.

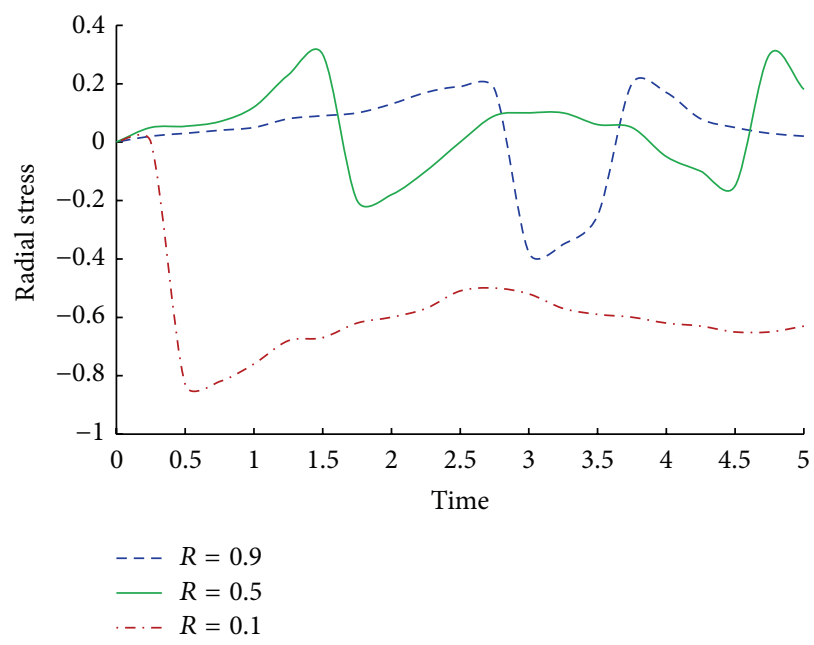

FIgURE 7: Impact radial stress variations of external wall with time $(s=4)$.

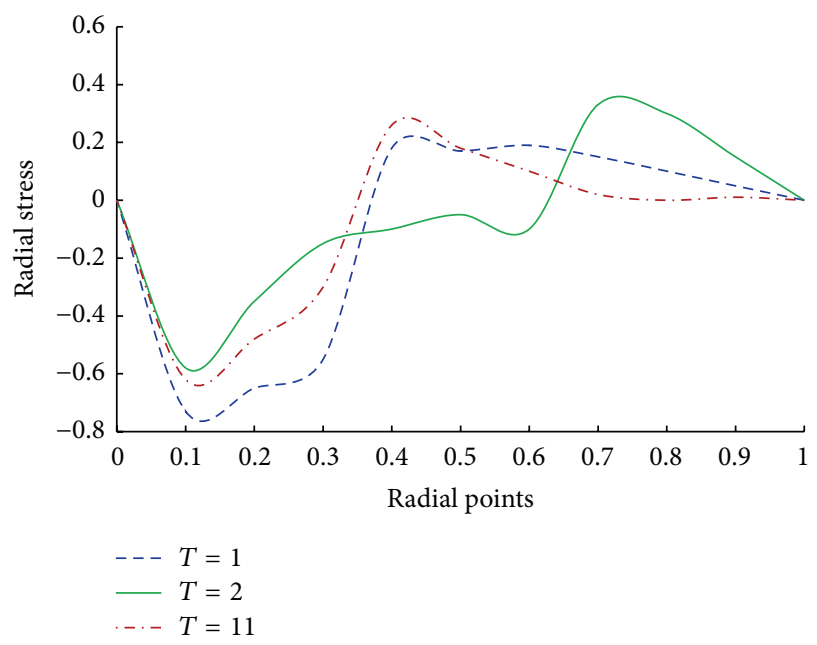

FIGURE 8: Impact radial stress variations of external wall with radius $(s=4)$. 


\section{Discussions and Conclusions}

A Green's function method was conducted in this research to discuss the dynamic stress distribution of thick-walled spherical shell under an isotropic impact load on spherical internal and external surface. Results obtained by the proposed Green's function method show that radial dynamic stress values have a remarkable change near both internal and external walls. For an isotropic impact load on spherical internal shell, the maximum compressive stress occurs near the impact wall, while the maximum tensile stress is near the other side. For an isotropic impact load on spherical external shell, the maximum compression stress is always near the inner wall. Results also verify that the proposed Green's function method can be conveniently used to analyze the dynamic stress distribution of thick-walled spherical shell under an isotropic impact load.

\section{Conflict of Interests}

The authors declare that there is no conflict of interests regarding the publication of this paper.

\section{Acknowledgments}

This research was supported by Henan Science and Technology Research Planning Project (Grant no. 152102310318) and Doctoral Fund of Anyang Normal University (Grant no. 320034).

\section{References}

[1] H. Hu, "On the general theory of elasticity for a spherically isotropic medium," Acta Physica Sinica, vol. 10, no. 1, pp. 57-70, 1954.

[2] Y. Zongda and F. Yibin, "Solution of axisymmetric thick spherical shell by finite spherical layer method," Computers \& Structures, vol. 30, no. 4, pp. 923-927, 1988.

[3] N. K. Gupta and Venkatesh, "Experimental and numerical studies of dynamic axial compression of thin walled spherical shells," International Journal of Impact Engineering, vol. 30, no. 8-9, pp. 1225-1240, 2004.

[4] A. B. Kiselev, "Analytic solutions of problems of the adiabatic compression of thick-walled spherical and cylindrical shells made of incompressible viscoplastic material," Journal of Applied Mathematics and Mechanics, vol. 76, no. 4, pp. 489-492, 2012.

[5] G. DeBotton, R. Bustamante, and A. Dorfmann, "Axisymmetric bifurcations of thick spherical shells under inflation and compression," International Journal of Solids and Structures, vol. 50, no. 2, pp. 403-413, 2013.

[6] Y. G. Pronina, "Analytical solution for decelerated mechanochemical corrosion of pressurized elastic-perfectly plastic thickwalled spheres," Corrosion Science, vol. 90, no. 4, pp. 161-167, 2015.

[7] P. C. Chou and H. A. Koenig, "A unified approach to cylindrical and spherical elastic waves by method of characteristics," Journal of Applied Mechanics, vol. 33, no. 1, pp. 159-167, 1966.
[8] G. Cinelli, "Dynamic vibrations and stresses in elastic cylinders and spheres," Journal of Applied Mechanics, vol. 33, no. 4, pp. 825-830, 1966.

[9] Y.-H. Pao and A. N. Ceranoglu, "Determination of transient responses of a thick-walled spherical shell by the ray theory," Journal of Applied Mechanics, vol. 45, no. 1, pp. 114-122, 1978.

[10] G. Z. Voyiadjis and P. Woelke, "A refined theory for thick spherical shells," International Journal of Solids and Structures, vol. 41, no. 14, pp. 3747-3769, 2004.

[11] S. Wu, Y. Qu, and H. Hua, "Vibration characteristics of a spherical-cylindrical-spherical shell by a domain decomposition method," Mechanics Research Communications, vol. 49, pp. 17-26, 2013.

[12] H. Ding, Y. Ren, D. Zou, and W. Chen, "Displacement method of elasticity problems in spherically isotropic media," Acta Mechanica Sinica, vol. 26, no. 2, pp. 186-197, 1994.

[13] W. Da, "Stress wave propagation in elastic solid sphere subjected to impact load," Journal of Henan University of Science and Technology, vol. 25, no. 5, pp. 85-88, 2004.

[14] D. W. Mackowski, "A general superposition solution for electromagnetic scattering by multiple spherical domains of optically active media," Journal of Quantitative Spectroscopy and Radiative Transfer, vol. 133, pp. 264-270, 2014.

[15] G. Carbone and L. Mangialardi, "Analysis of the adhesive contact of confined layers by using a Green's function approach," Journal of the Mechanics and Physics of Solids, vol. 56, no. 2, pp. 684-706, 2008.

[16] J. Lv, X. Yuan, and J. Xiao, "Green function method for solving elastic solid sphere under an isotropic impact load," Journal of Zhengzhou University, vol. 45, no. 4, pp. 109-113, 2013.

[17] R. Dill, V. Klemann, Z. Martinec, and M. Tesauro, "Applying local Green's functions to study the influence of the crustal structure on hydrological loading displacements," Journal of Geodynamics, vol. 88, pp. 14-22, 2015.

[18] J. Lv and D. Ji, "Solution of characteristic equation in eigenvalue problems of spherical Bessel equation by using Matlab algorithm," Journal of Lanzhou University of Technology, vol. 33, no. 3, pp. 152-155, 2007.

[19] Z. Wang, Equations of Mathematical Physics and Special Function, Tsinghua University Press, 1991.

[20] D. D. Achenbach, Wave Propagation in Elastic Solids, North Holland, 1973. 


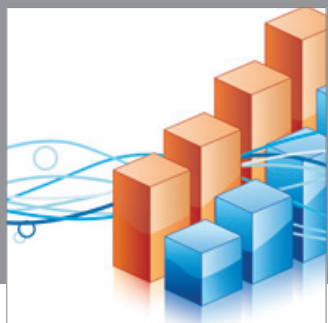

Advances in

Operations Research

mansans

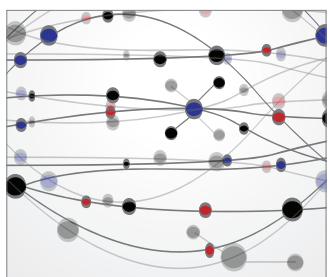

The Scientific World Journal
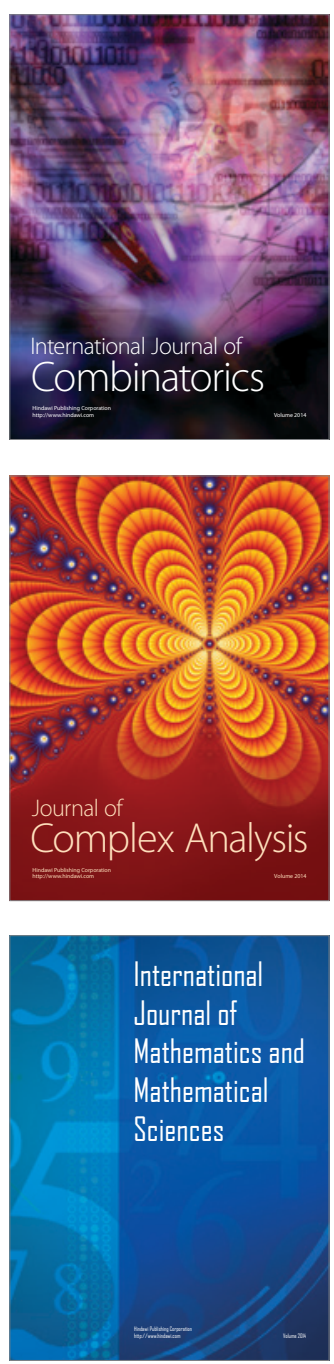
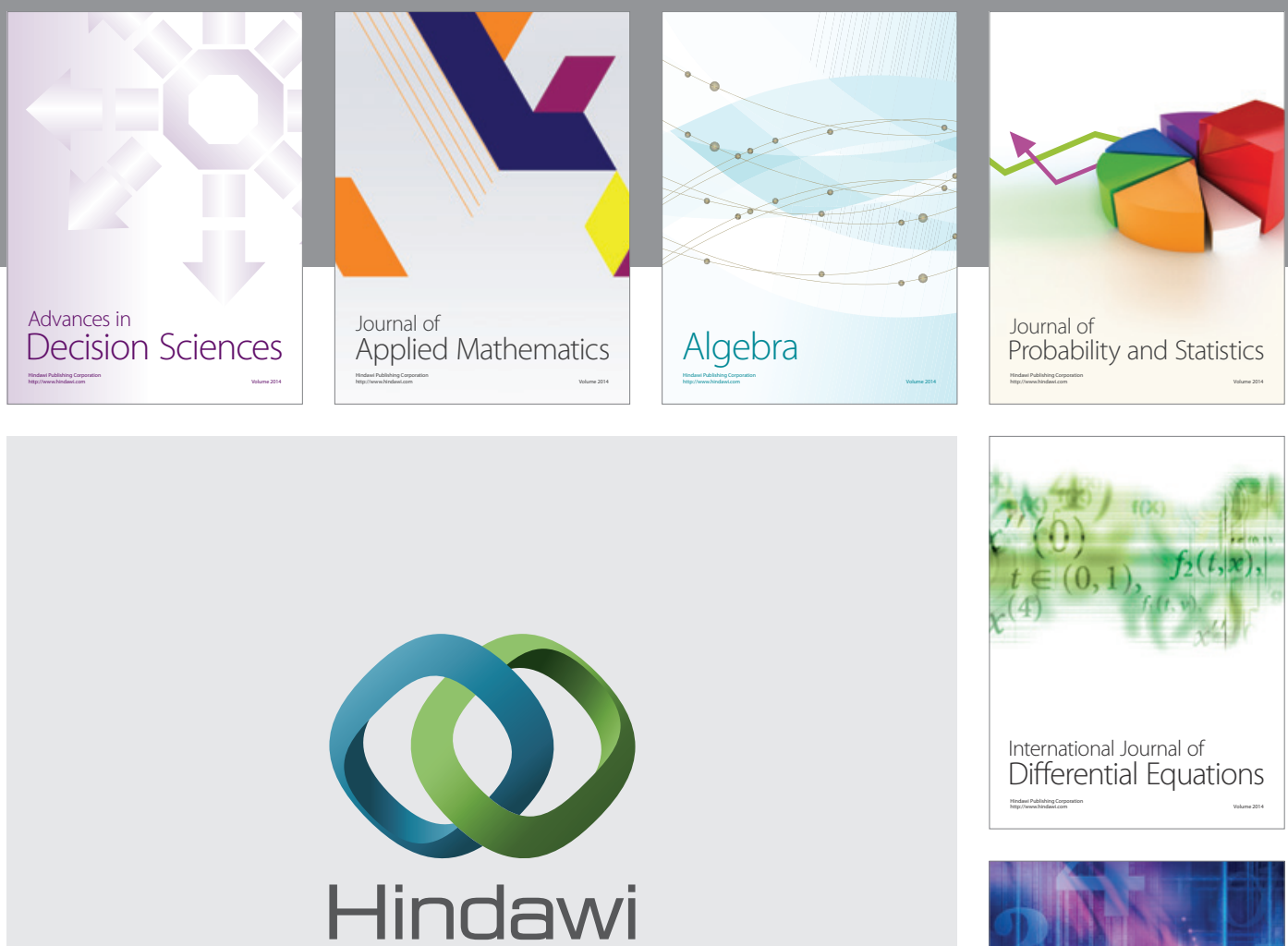

Submit your manuscripts at http://www.hindawi.com
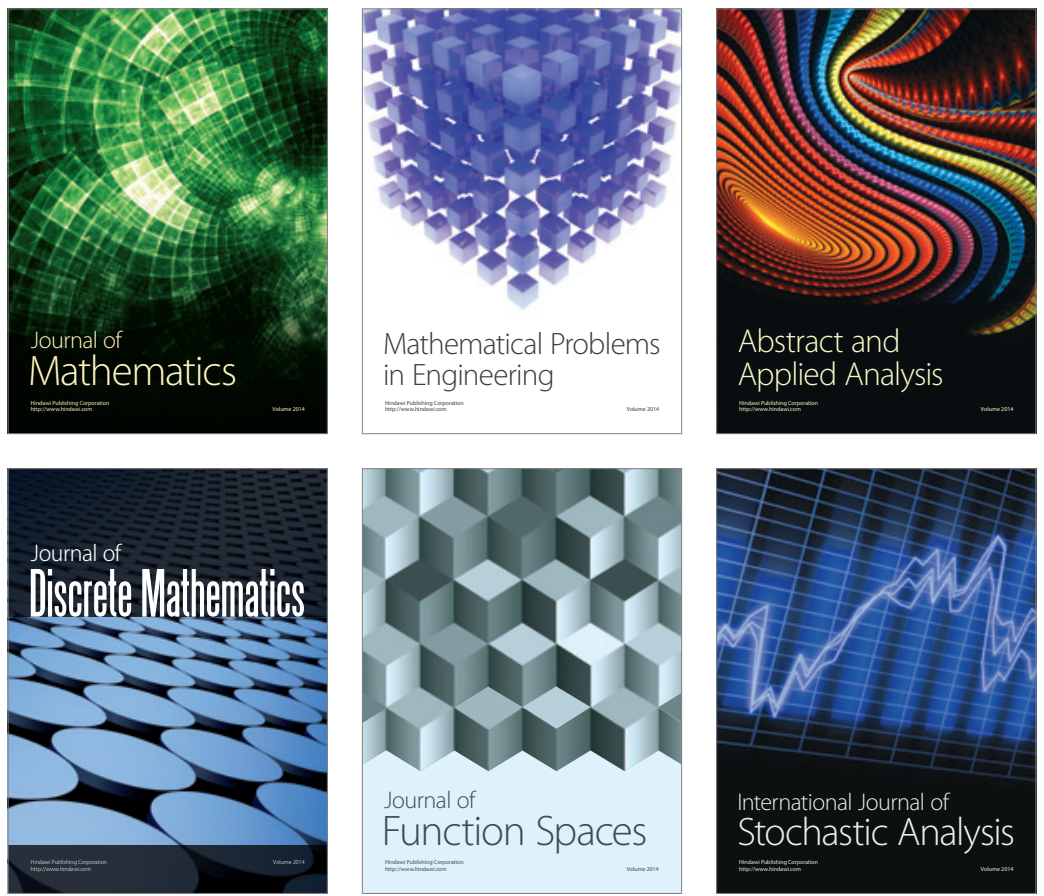

Journal of

Function Spaces

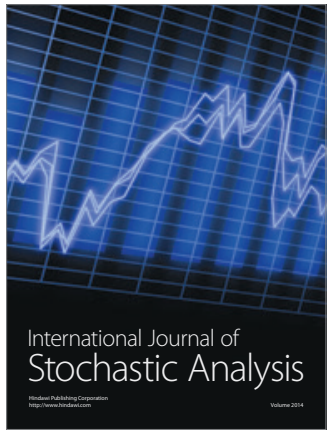

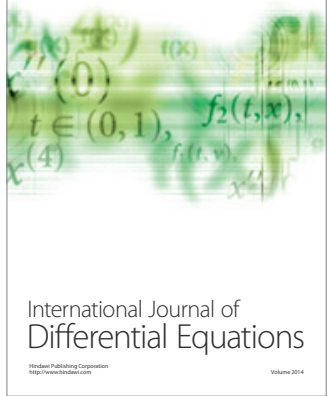
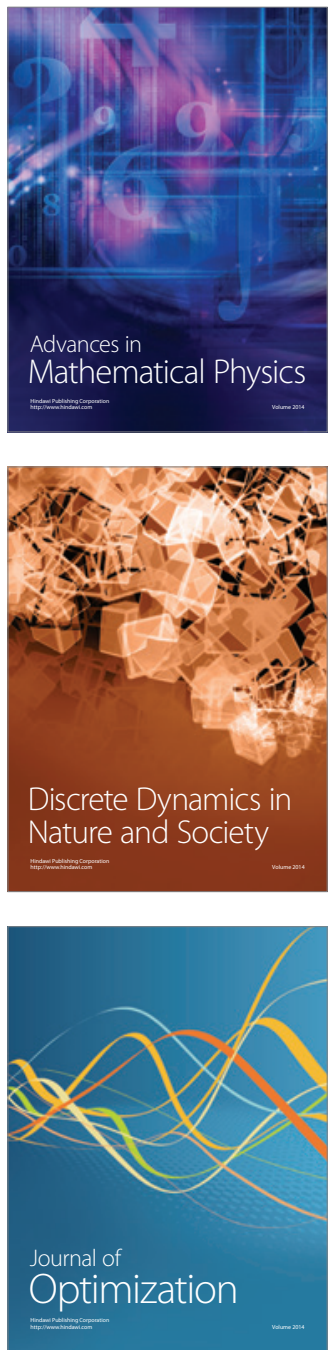